\title{
OPEN Fecal bacteria and metabolite responses to dietary lysozyme in a sow model from late gestation until lactation
}

\author{
Shengyu Xu $\mathbb{1}^{1,4^{*}}$, Jiankai Shi ${ }^{1,4}$, Yanpeng Dong ${ }^{1}$, Zimei $\mathrm{Li}^{1}$, Xiaoling Wu ${ }^{1}$, Yan Lin ${ }^{1}$, \\ Lianqiang Che ${ }^{1}$, Jian $\mathrm{Li}^{1}$, Bin Feng ${ }^{1}$, Zhengfeng Fang ${ }^{1}$, Yong Zhuo ${ }^{1}$, Jianping Wang ${ }^{1}$, De Wu ${ }^{1}$, \\ Zhihua Ren ${ }^{2}$ \& Yanping Shen ${ }^{3}$
}

Lysozyme (LZM) is a natural anti-bacterial protein that is found in the saliva, tears and milk of all mammals including humans. Its anti-bacterial properties result from the ability to cleave bacterial cell walls, causing bacterial death. The current study was conducted to investigate the effects of dietary LZM on fecal microbial composition and variation in metabolites in sow. The addition of LZM decreased the fecal short-chain fatty acids (SCFAs). Zonulin and endotoxin in the serum, and feces, were decreased with lysozyme supplementation. Furthermore, fecal concentrations of lipocalin-2 and the pro-inflammatory cytokine TNF- $\alpha$ were also decreased while the anti-inflammatory cytokine IL-10 was increased by lysozyme supplementation. $16 \mathrm{~S}$ rRNA gene sequencing of the $\mathrm{V} 3-\mathrm{V} 4$ region suggested that fecal microbial levels changed at different taxonomic levels with the addition of LZM. Representative changes included the reduction of diversity between sows, decreased Bacteroidetes, Actinobacteria, Tenericutes and Spirochaetes during lactation as well as an increase in Lactobacillus. These findings suggest that dietary lysozyme supplementation from late gestation to lactation promote microbial changes, which would potentially be the mechanisms by which maternal metabolites and inflammatory status was altered after LZM supplementation.

The perinatal period is a key window for mother and offspring which brings great changes in maternal metabolism, hormones, immunity and microbiota ${ }^{1,2}$. Microbiota remodeling during the perinatal period may be a positive process enabling the mother to support their own health, as well as that of their offspring. Gut microbiota play a key role in the metabolism of nutrients, immune function, gut hormone secretion and provide protection from pathogens ${ }^{2-5}$. At the same time, microbial flora is regulated by nutrients as well as the immunological and metabolic statuses of the host. During farrowing the substantial physiological changes associated can lead to inflammatory reactions in sows ${ }^{6}$. Furthermore, studies have found an increase in pro-inflammatory factors during early lactation that was significantly higher than during early pregnancy or during the late stages of lactation ${ }^{7,8}$.

Lysozyme has been shown to be at high levels $(400 \mathrm{mg} / \mathrm{L})$ in human milk compared to the milk of other species $^{\text {? }}$. Previous studies have found that lysozyme is beneficial to establish the bifidobacteria- and lactobacilli-rich intestinal microflora in breastfed infants, which may promote maturation of the intestinal tract ${ }^{10-13}$. Lysozyme is also involved in modulating the inflammatory response ${ }^{14,15}$. As a natural food and anti-infective substance, lysozyme has been used as an additive in the infant food and within the medical industry. In piglets it has been found that lysozyme (LZM), acting as an alternative to antibiotics, has beneficial effects on gut morphology ${ }^{16,17}$, immunity $^{18,19}$, intestinal flora ${ }^{19}$, and growth performance ${ }^{20,21}$. Results of our previous study suggested that adding LZM to the diet of sows' resulted in an increased average daily feed intake (ADFI) during lactation, shortened

\footnotetext{
${ }^{1}$ Animal Nutrition Institute, Sichuan Agricultural University, Key laboratory of Animal Disease-resistant Nutrition, Ministry of Education, Key laboratory of Animal Disease-resistant Nutrition, Ministry of Agriculture and Rural Affairs, Key laboratory of Animal Disease-resistant Nutrition, Sichuan Province, Chengdu, 611130, Sichuan, P. R. China. ${ }^{2}$ College of Veterinary Medicine, Sichuan Province Key Laboratory of Animal Disease and Human Health, Key Laboratory of Environmental Hazard and Human Health of Sichuan Province, Sichuan Agricultural University, Chengdu, 611130, P. R. China. ${ }^{3}$ Shanghai Longyou Biotechnology Co, Ltd, Shanghai, 200000, P. R. China. ${ }^{4}$ These authors contributed equally: Shengyu Xu and Jiankai Shi. *email: shengyu_x@hotmail.com
} 


\begin{tabular}{|c|c|c|c|c|}
\hline \multirow[b]{2}{*}{ Item } & \multicolumn{3}{|l|}{ Treatment } & \multirow[b]{2}{*}{$P$-value } \\
\hline & \begin{tabular}{|l|} 
Control \\
\end{tabular} & LZM 150 & LZM 300 & \\
\hline \multicolumn{5}{|l|}{ d 1 of lactation } \\
\hline $\mathrm{pH}$ value & $7.27 \pm 0.21$ & $7.72 \pm 0.44$ & $7.52 \pm 0.29$ & 0.08 \\
\hline Acetate, $\mathrm{mg} / \mathrm{g}$ & $6.64 \pm 2.31$ & $5.24 \pm 2.00$ & $5.33 \pm 2.00$ & 0.43 \\
\hline Propionate, $\mathrm{mg} / \mathrm{g}$ & $2.29 \pm 0.89$ & $1.67 \pm 0.74$ & $1.41 \pm 0.62$ & 0.13 \\
\hline Butyrate, $\mathrm{mg} / \mathrm{g}$ & $1.04 \pm 0.54$ & $0.58 \pm 0.31$ & $1.02 \pm 0.31$ & 0.07 \\
\hline Total SCFAs, mg/g & $9.98 \pm 3.69$ & $7.49 \pm 2.96$ & $7.77 \pm 2.57$ & 0.31 \\
\hline \multicolumn{5}{|l|}{ d 7 of lactation } \\
\hline $\mathrm{pH}$ value & $6.93 \pm 0.35^{\mathrm{b}}$ & $7.66 \pm 0.32^{\mathrm{a}}$ & $7.37 \pm 0.28^{\mathrm{a}}$ & $<0.01$ \\
\hline Acetate, $\mathrm{mg} / \mathrm{g}$ & $12.25 \pm 1.12^{\mathrm{a}}$ & $7.73 \pm 0.93^{\mathrm{b}}$ & $7.78 \pm 1.90^{\mathrm{b}}$ & $<0.01$ \\
\hline Propionate, $\mathrm{mg} / \mathrm{g}$ & $3.40 \pm 0.53$ & $2.46 \pm 0.21$ & $2.54 \pm 0.96$ & 0.06 \\
\hline Butyrate, mg/g & $1.79 \pm 0.32^{\mathrm{a}}$ & $1.02 \pm 0.22^{\mathrm{b}}$ & $1.26 \pm 0.45^{\mathrm{b}}$ & $<0.01$ \\
\hline Total SCFAs, mg/g & $17.44 \pm 1.43^{\mathrm{a}}$ & $11.21 \pm 1.23^{\mathrm{b}}$ & $11.59 \pm 3.21^{\mathrm{b}}$ & $<0.01$ \\
\hline \multicolumn{5}{|l|}{ d 21 of lactation } \\
\hline $\mathrm{pH}$ value & $7.04 \pm 0.33^{b}$ & $7.64 \pm 0.32^{\mathrm{a}}$ & $7.35 \pm 0.48^{\mathrm{a}}$ & 0.02 \\
\hline Acetate, $\mathrm{mg} / \mathrm{g}$ & $15.50 \pm 5.52$ & $10.82 \pm 4.29$ & $10.98 \pm 3.47$ & 0.09 \\
\hline Propionate, $\mathrm{mg} / \mathrm{g}$ & $5.26 \pm 2.03$ & $3.91 \pm 2.17$ & $3.77 \pm 1.62$ & 0.27 \\
\hline Butyrate, $\mathrm{mg} / \mathrm{g}$ & $2.69 \pm 1.05^{\mathrm{a}}$ & $1.44 \pm 0.78^{\mathrm{b}}$ & $1.46 \pm 0.65^{\mathrm{b}}$ & 0.01 \\
\hline Total SCFAs, mg/g & $23.45 \pm 8.48$ & $16.17 \pm 7.14$ & $16.22 \pm 5.61$ & 0.09 \\
\hline
\end{tabular}

Table 1. Effects of feeding sows diets supplemented with lysozyme on fecal SCFAs concentrations of sows. Data are expressed as mean \pm SEM. Sows were regarded as the experimental units, $n=10$ for each treatment. LZM $150=$ control diet + lysozyme $150 \mathrm{mg} / \mathrm{kg}$, LZM $300=$ control diet + lysozyme $300 \mathrm{mg} / \mathrm{kg}$. SCFAs is the sum of acetate, propionate, and butyrate. a,bWithin a row, means with different superscripts are different $(\mathrm{P}<0.05)$.

weaning-to-estrus interval (WEI), and improved sow and offspring health as indicated by altered inflammatory cytokines and a reduction in the piglets' diarrhea rate ${ }^{22}$. However, there remains little knowledge of the maternal diversity of gut microbiota following lysozyme supplementation during the perinatal period. As a 1, 4- $\beta$-N-acetylmuramidase, lysozyme cleaves the $\beta$-1,4-glycosidic bond between the $\mathrm{N}$-acetylmuramic acid and $\mathrm{N}$-acetylglucosamine residues of the bacterial peptidoglycan, resulting in an incomplete cell membrane and leading to cell death ${ }^{23}$. This suggests that dietary lysozyme would lead to variation in the microbiota in the gut, which has been confirmed in piglets and infants ${ }^{13,19,24}$. Therefore, we investigated the effect on the maternal microbiota following lysozyme supplementation.

Thus, in this study we investigated the effects of adding lysozyme to maternal diets on gut permeability, gut inflammation, gut microbial and metabolite composition from the period of late gestation to lactation in a sow model.

\section{Results}

Fecal changes in $\mathrm{pH}$ and concentration of short-chain fatty acids (SCFAs). As shown in Table 1, LZM 150 or LZM 300 had no effect on the $\mathrm{pH}$ and concentration of SCFAs, in sow fecal material, on day 1 of lactation. In contrast, both LZM diets increased $\mathrm{pH}$ values on days 7 and 21 of lactation $(P<0.05)$ when compared with the control diet. However, no difference was found between the two LZM treatments. Sows fed with the LZM 150 and LZM 300 diets showed decreased $(P<0.05)$ fecal levels of acetate, butyrate and total SCFAs on day 7 of lactation and decreased fecal levels of butyrate on day 21 of lactation when compared to sows from the control diet group. Interestingly, concentrations of total and individual SCFA increased linearly from day 1 to day 21 of lactation (Fig. S1).

Difference in gut permeability and endotoxin concentrations. As a biomarker for gut permeability serum zonulin was measured. As can be seen in Fig. 1A1, no differences were found between the treatments in terms of the serum levels of zonulin on day 1 of lactation. Sows which were fed the LZM 150 and LZM 300 diets both showed decreased zonulin in their serum on days 7 and 21 of lactation (Fig. 1A2,A3, $P<0.05$ ). This indicates that sows' gut permeability was decreased by the LZM treatment. In addition, when compared with the control groups, both LZM 150 and LZM 300 treatments decreased (Fig. 1B2,B3,C2,C3, $P<0.05$ ) the serum and fecal concentrations of endotoxin on days 7 and 21 of lactation, although no difference was found on day 1 of lactation (Fig. 1B1,C1).

Inflammation state of the sows' intestinal mucosal epithelium. Four biomarkers of inflammation were measured to reflect effects of LZM on the inflammatory response in sows' intestines. As can be seen in Fig. 2, concentrations of fecal lipocalin-2 and TNF- $\alpha$ (pro-inflammatory cytokines) were decreased on days 7 and 21 of lactation in sows which had been fed the LZM 150 and LZM 300 diets (Fig. 2A,B). However, no significant differences were found in fecal IL-6 between the treatments (Fig. 2C1-3). In contrast, concentrations of IL-10 (anti-inflammatory cytokine) increased on days 7 and 21 of lactation for the sows on the LZM 150 diets (Fig. 2D1-3). 

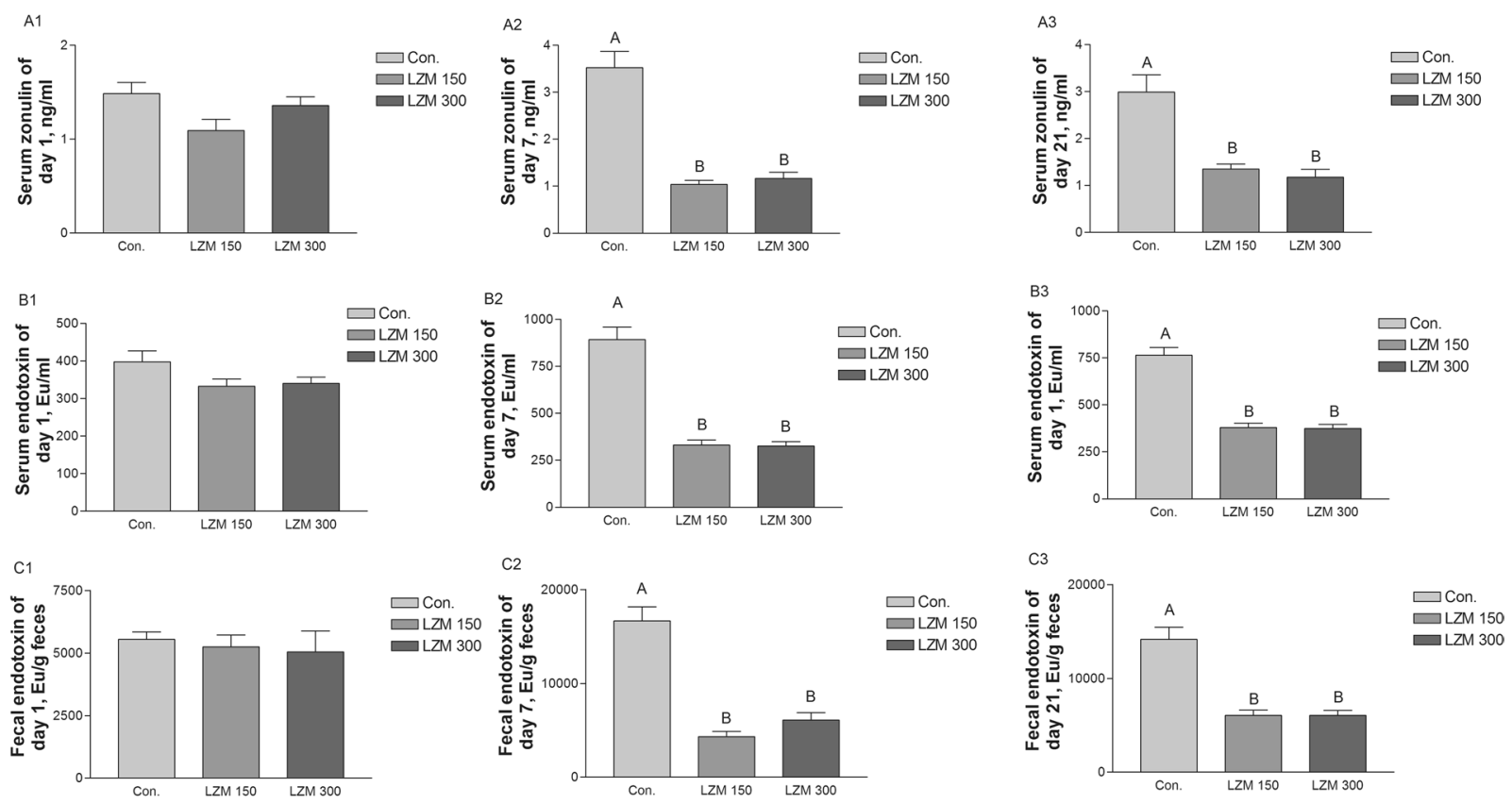

Figure 1. Effects of feeding sows diets supplemented with lysozyme on the serum concentrations of zonulin (A1-3) and endotoxin (B1-3) and fecal concentrations of endotoxin (C1-3). Data are presented as means $\pm \operatorname{SEM}(n=8)$. Con. $=$ control, LZM $150=$ control diet + lysozyme $150 \mathrm{mg} / \mathrm{kg}, \mathrm{LZM} 300=$ control diet + lysozyme $300 \mathrm{mg} / \mathrm{kg}$. A, B, significant effect of treatment (values with different uppercase letters are significantly different, $P<0.01)$.

Microbial diversity in sows' feces. A total of 54 fecal samples were subjected to $16 \mathrm{~S}$ rRNA gene sequencing. Average raw reads, average effective tags and average operational taxonomic units (OTUs) for each treatment were shown in Supplementary Table S1. A set of 1,272 OTUs existed in all treatments and were thus defined as core OTUs (Fig. S2). These comprised $71.5 \%$ of the total number of OTUs, whereas 44, 37, 52, 135, 47, 105, 72, 56 and 57 OTUs were uniquely identified at Con. d1, LZM 150 d1, LZM 300 d1, Con. d7, LZM 150 d7, LZM 300 d7, Con. d21, LZM $150 \mathrm{~d} 21$ and LZM $300 \mathrm{~d} 21$, respectively (Fig. S2). To determine the bacterial diversity, the alpha and beta diversity of the fecal microbiota were assessed. We then compared the richness (observed species and Chao 1 index) and diversity (Shannon index) indices for the alpha diversity. As demonstrated in Table 2, LZM 300 diets decreased the observed species and Chao 1 index at day 21 of lactation. The lactation stage had a significant effect on fecal microbial community richness, with a Chao 1 index on day 1 of lactation being lower than at other stages $(P<0.01)$. A decrease in Shannon index (fecal microbial community diversity) with LZM supplementation at $300 \mathrm{mg} / \mathrm{kg}$ was found on day 7 and 21 of lactation $(P<0.01)$. For the analysis of beta diversity, the relationships among Control, LZM 150 and LZM 300 on day 1,7 and 21 of lactation in the gut microbiome were examined by principal component analysis. The gut microbiota of sows showed obvious segregation in the different treatments, especially in treatment LZM300 d21 (Fig. 3) based on weighted UniFrac distance. The PERMANOVA analysis found that the bacterial community structure was significantly $(P<0.05)$ different after LZM supplementation (in Supplementary Table S2) based on the Bray-Curtis distance measures. This further indicated that lysozyme supplementation significantly affected the diversity of bacterial in the fecal matter.

Changes of gut microbiota composition by lysozyme in sows. The relative abundances of fecal microbiota at the phylum and genus levels for all samples during lactation are displayed in Supplementary Fig. S3. The top six dominated phyla are Firmicutes (73.96\%), Bacteroidetes (11.95\%), Proteobacteria (6.54\%), Tenericutes $(2.50 \%)$, Euryarchaeota $(1.62 \%)$ and Spirochaetes $(0.98 \%)$. At the genus level, Clostridium_sensu_stricto_1 (7.74\%), Ruminococcaceae_NK4A214_group (6.67\%), Ruminococcaceae_UCG-002 (5.72\%), [Eubacterium] _coprostanoligenes_group (5.41\%), Ruminococcaceae_UCG-005 (4.28\%), Streptococcus (3.74\%), Lactobacillus (3.19\%), Escherichia-Shigella (2.55\%), Methanobrevibacter (1.60\%) and Bacillus (1.30\%) were the 10 dominating genera.

Differences in the fecal microbiota among the three treatments at the phylum and genus levels were identified on day 1, 7 and 21 of lactation. Sows which had been fed the LZM 300 diets showed decreased relative abundance of Bacteroidetes and Actinobacteria in the gut at the phylum level on days 7 and 21 of lactation (Fig. 4A,B). In addition, sows fed LZM 150 diets showed decreased relative levels of Tenericutes and Spirochaetes in the gut on days 1 and 7 of lactation (Fig. 4C,D). However, when compared with the control group sows which had been fed the LZM 300 diets had increased relative levels of Proteobacteria on day 1 of lactation (Fig. 4E). The relative abundance of Euryarchaeota was decreased in the LZM 300 treatment group compared with the LZM 150 treatment group on day 21 of lactation (Fig. 4F). At the genus level, 23 genera relative abundances changed across the different treatments on day 1, 7 and 21 of lactation (Table 3). Sows which had been fed the LZM 150 and LZM 300 diets 

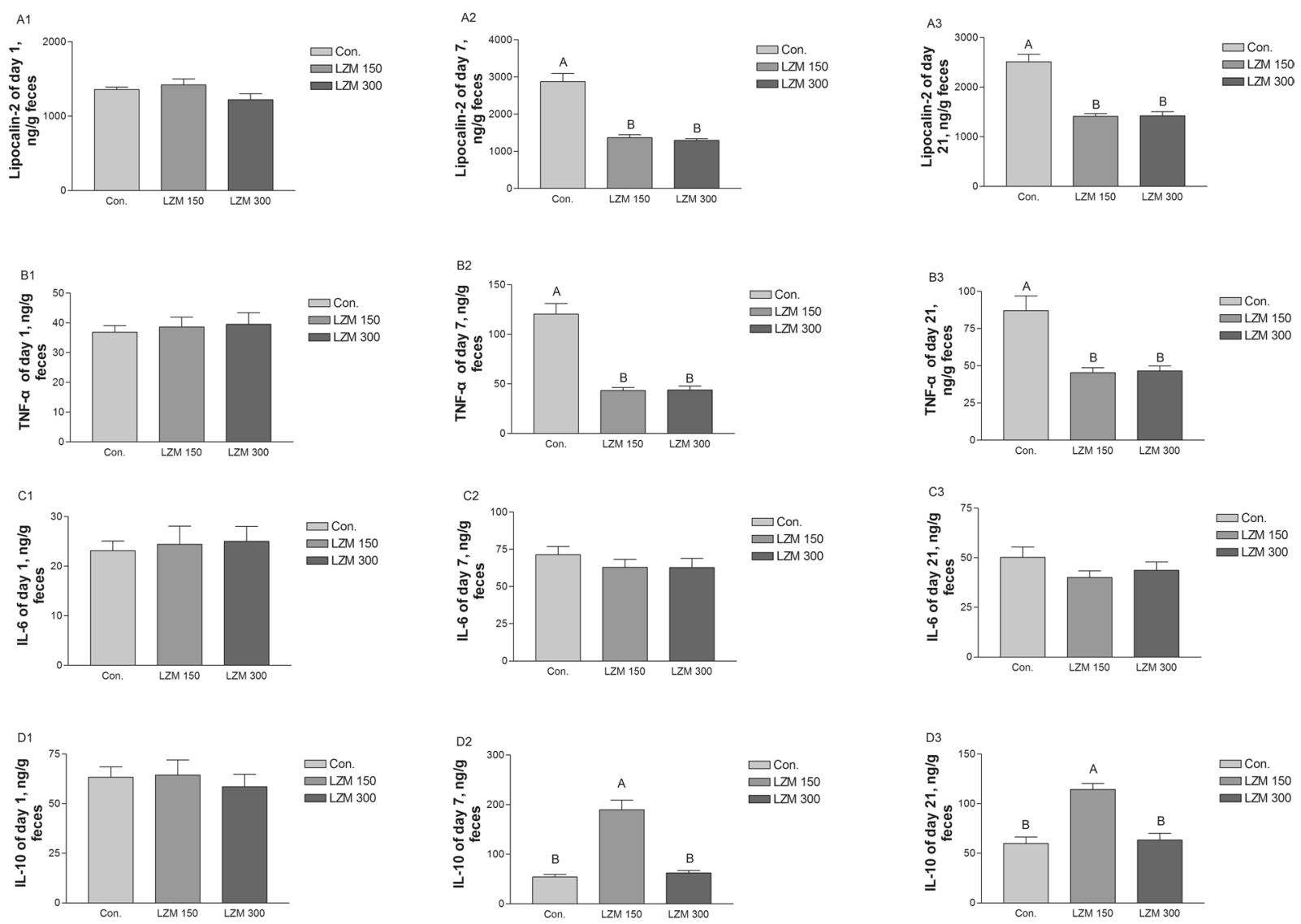

Figure 2. Effects of feeding sows diets supplemented with lysozyme on the serum concentrations of zonulin (A1-3) and endotoxin (B1-3) and fecal concentrations of endotoxin. Data are presented as means \pm SEM $(n=8)$. TNF- $\alpha=$ tumor necrosis factor- $\alpha$, IL-6 = interleukin-6, IL-10 = interleukin-10. Con. = control, LZM $150=$ control diet + lysozyme $150 \mathrm{mg} / \mathrm{kg}$, LZM $300=$ control diet + lysozyme $300 \mathrm{mg} / \mathrm{kg}$. A, B, significant effect of treatment (values with different uppercase letters are significantly different, $P<0.01$ ).

\begin{tabular}{|c|c|c|c|c|c|c|c|}
\hline \multirow[b]{2}{*}{ Item } & \multirow[b]{2}{*}{ time } & \multirow[b]{2}{*}{ CON } & \multirow{2}{*}{\begin{tabular}{|l|} 
Treatment \\
LZM 150 \\
\end{tabular}} & \multirow[b]{2}{*}{ LZM 300} & \multicolumn{3}{|c|}{$P$-value } \\
\hline & & & & & diet & time & diet*time \\
\hline \multirow{3}{*}{ Observed species } & d 1 & $1463.33 \pm 186.88$ & $1361.50 \pm 81.21$ & $1531.67 \pm 155.56^{*}$ & \multirow{3}{*}{0.19} & \multirow{3}{*}{0.02} & \multirow{3}{*}{$<0.01$} \\
\hline & $\mathrm{d} 7$ & $1480.67 \pm 268.49$ & $1733.50 \pm 174.18^{*}$ & $1691.67 \pm 132.01^{*}$ & & & \\
\hline & d 21 & $1744.17 \pm 139.69^{\mathrm{a}^{*}}$ & $1628.33 \pm 88.08^{\mathrm{a}^{*}}$ & $1201.00 \pm 170.23^{\mathrm{b}}$ & & & \\
\hline \multirow{3}{*}{ Chao 1} & d 1 & $1655.92 \pm 202.79$ & $1541.86 \pm 121.58$ & $1718.32 \pm 166.63$ & \multirow{3}{*}{0.11} & \multirow{3}{*}{$<0.01$} & \multirow{3}{*}{$<0.01$} \\
\hline & d 7 & $1672.27 \pm 272.25$ & $2072.34 \pm 325.19^{*}$ & $1906.23 \pm 106.26^{*}$ & & & \\
\hline & d 21 & $2034.73 \pm 153.27^{\mathrm{a}^{*}}$ & $1909.28 \pm 131.70^{\mathrm{a}^{*}}$ & $1359.10 \pm 191.20^{\mathrm{b}}$ & & & \\
\hline \multirow{3}{*}{ Shannon } & d 1 & $7.50 \pm 0.25^{\mathrm{a}}$ & $7.32 \pm 0.12^{\mathrm{b}}$ & $7.58 \pm 0.05^{\mathrm{a}^{\mathrm{a}}}$ & \multirow{3}{*}{$<0.01$} & \multirow{3}{*}{$<0.01$} & \multirow{3}{*}{$<0.001$} \\
\hline & d 7 & $7.43 \pm 0.21^{\mathrm{a}}$ & $7.52 \pm 0.28^{\mathrm{a}}$ & $7.15 \pm 0.53^{\mathrm{b}}$ & & & \\
\hline & d 21 & $7.58 \pm 0.12^{\mathrm{a}}$ & $7.31 \pm 0.17^{\mathrm{ab}}$ & $6.62 \pm 0.17^{\mathrm{b}}$ & & & \\
\hline
\end{tabular}

Table 2. Effects of feeding sows diets supplemented with lysozyme on microbiota alpha diversity index of sows. Data are expressed as mean \pm SEM. Sows were regarded as the experimental units, $n=6$ for each treatment. LZM $150=$ control diet + lysozyme $150 \mathrm{mg} / \mathrm{kg}$, LZM $300=$ control diet + lysozyme $300 \mathrm{mg} / \mathrm{kg}$. a,bWithin a row, means with different superscripts are different $(\mathrm{P}<0.05)$. ${ }^{*}$ Within a coloum in the same index at different day, means with asterisk denotes different $(\mathrm{P}<0.05)$.

had decreased relative levels of Ruminococcaceae_UCG-005 on day 1 of lactation, decreased relative abundances of Parabacteroides, Prevotella_1, Cellulosilyticum and Succinivibrio on day 7 of lactation, and increased relative abundances of Lactobacillus and Ruminococcaceae_UCG-013 on day 21 of lactation. However, sows fed the LZM 150 diets had increased relative abundance of Methanobrevibacter and Ruminococcaceae_UCG-009 on day 21 of lactation. Additionally, the LZM 300 treatment increased the relative abundances of Romboutsia and Acinetobacter on day 1 of lactation, Ruminococcaceae_UCG-009 on day 7 of lactation, and Enterococcus, Staphylococcus on day 21 compared with the control group. Sows which had been fed LZM 300 diets had decreased relative abundances 


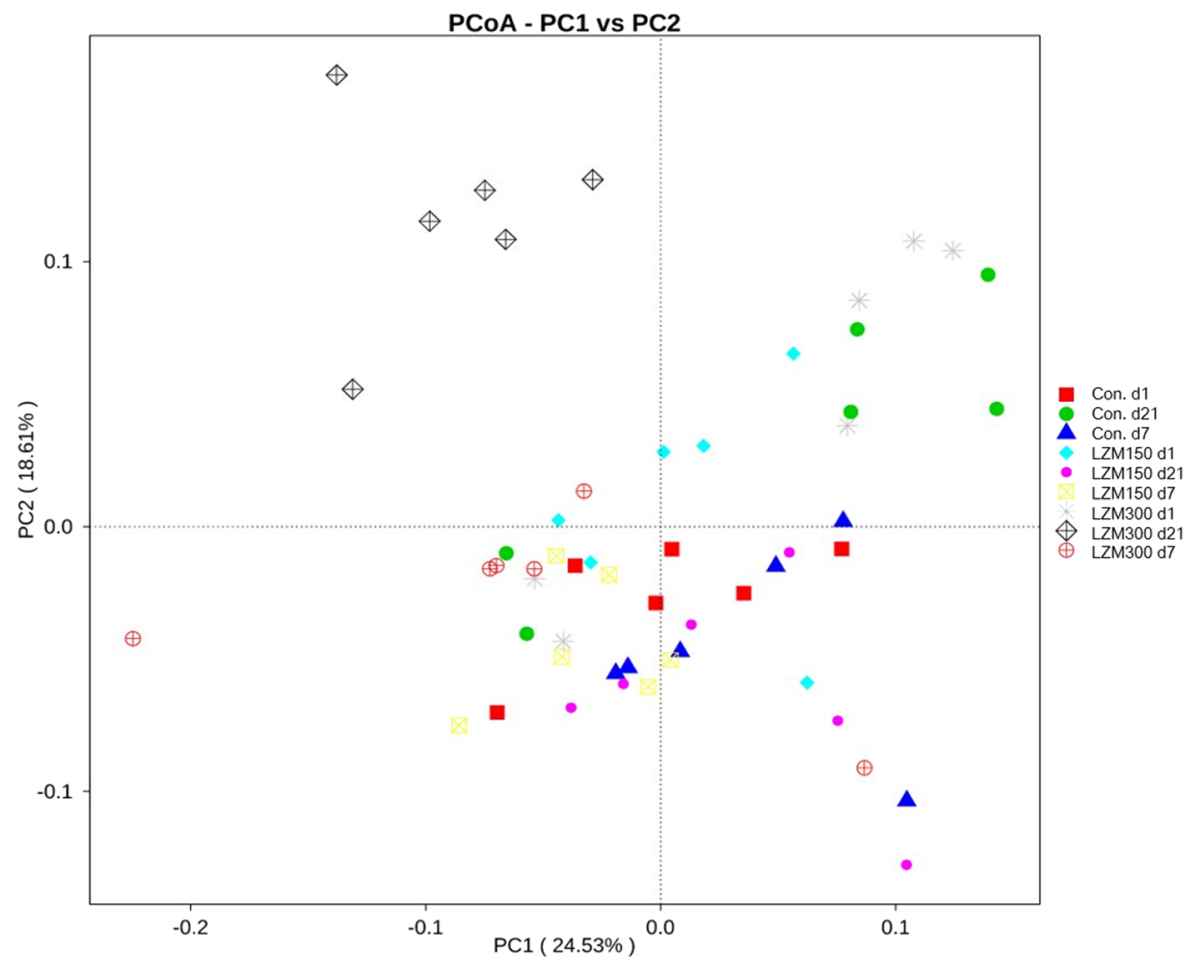

Figure 3. Comparison of the gut microbiota composition among treatments. Principal coordinate analysis to visualize the weighted UniFrac distances of fecal samples from individual sow. Con.d $1=$ control at day 1 of lactation, Con.d7 = control at day 7 of lactation, Con.d7 = control at day 21 of lactation, LZM $150 \mathrm{~d} 1=$ control diet + lysozyme $150 \mathrm{mg} / \mathrm{kg}$ at day 1 of lactation, LZM $150 \mathrm{~d} 7=$ control diet + lysozyme $150 \mathrm{mg} / \mathrm{kg}$ at day 7 of lactation, LZM $150 \mathrm{~d} 21$ = control diet + lysozyme $150 \mathrm{mg} / \mathrm{kg}$ at day 21 of lactation, LZM $300 \mathrm{~d} 1=$ control diet + lysozyme $300 \mathrm{mg} / \mathrm{kg}$ at day 1 of lactation, LZM $300 \mathrm{~d} 7=$ control diet + lysozyme $300 \mathrm{mg} / \mathrm{kg}$ at day 7 of lactation, LZM $300 \mathrm{~d} 21$ = control diet + lysozyme $300 \mathrm{mg} / \mathrm{kg}$ at day 21 of lactation.

of Ruminococcaceae_UCG-010 (FDR P value = 0.052) on day 1 of lactation, Ruminococcaceae_UCG-014 on day 7 of lactation, and Terrisporobacter, Christensenellaceae_R-7_group, Oscillospira, Ruminococcaceae_UCG-010, Romboutsia on day 21 of lactation.

Based on multiple-taxonomic LEfSe analysis of day 21 of lactation, at the phylum level Euryarchaeota was enriched in sows from the LZM 150 group, and Proteobacteria was enriched in sows from the LZM 300 group (Fig. 5A,B). At the family level, Prevotellaceae and Moraxellaceae were enriched in sows from the control group, while Lachnospiraceae, Lactobacillaceae and Methanobacteriaceae were enriched in sows from the LZM 150 group. Enterobacteriaceae, Streptococcaceae, Enterococcaceae, Bacillaceae and Staphylococcaceae were enriched in sows from the LZM 300 group. At the genus level, Acinetobacter was enriched in sows from the control group, however Lactobacillus, Lachnospiraceae_XPB1014_group and Methanobacteriales were enriched in sows from the LZM 150 group, and Streptococcus, Enterococcus and bacillus were enriched in sows from the LZM 300 group. In addition, LEfSe analysis at days 1 and 7 of lactation demonstrated a difference of enriched bacteria (Fig. S4). Therefore, these results demonstrate that sows' gut microbiota composition is profoundly changed by the supplementation with LZM during lactation.

Correlations between the gut microbiota and metabolic related parameters in sows. As shown in Fig. 6, at the phylum level, Bacteroidetes, Euryarchaeota, Spirochaetes, Deferribacteres, and Aminicenantes were positively correlated with serum zonulin $(\mathrm{r}>0.50, P<0.05)$. However, Tenericutes was negatively correlated with serum zonulin $(\mathrm{r}=-0.51, P<0.01)$. Fusobacteria, Thaumarchaeota, Acidobacteria, Chloroflexi, Gemmatimonadetes, Nitrospirae, Thermomicrobia, FBP, Chlorobi, Aminicenantes, and Chlamydiae were positively correlated with fecal IL-6 $(\mathrm{r}>0.5, P<0.01)$. Thaumarchaeota, Acidobacteria, and Nitrospirae were positively correlated with fecal IL-10 $(\mathrm{r}<-0.50, P<0.05)$. Thaumarchaeota, Deinococcus. Thermus, Nitrospirae and Aminicenantes were positively correlated with acetate, propionate, butyrate and SCFAs $(\mathrm{r}>0.50, P<0.05)$. Cyanobacteria $(\mathrm{r}=-0.52, P<0.05)$ and Elusimicrobia $(\mathrm{r}=-0.59, P<0.01)$ were negatively correlated with acetate, propionate and SCFAs. In addition, Correlations analysis at the family and genus level were shown in Fig. S5. The correlation analysis of Bacteroidetes with serum zonulin and LZM at various taxonomic levels was interesting. A total of 4 genera in the Prevotellaceae family and 2 families within the phylum Bacteroidetes were found to be positively correlated $(\mathrm{r}>0.50, P<0.05)$ with the serum zonulin, and their abundance were decreased $(P<0.05)$ with the addition of LZM (Fig. S6). 

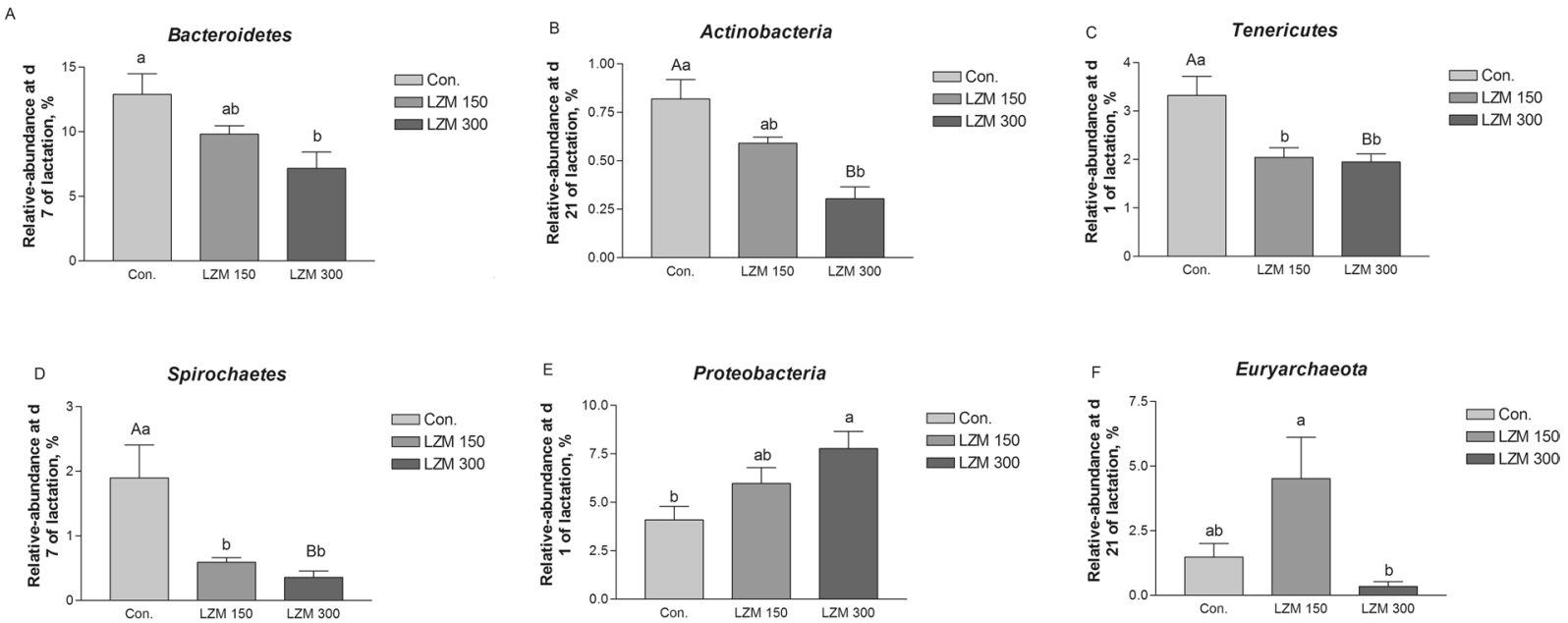

Figure 4. Changes in the six distinct bacterial phyla in sow gut of feeding sows diets supplemented with lysozyme. (A) Bacteroidetes, (B) Actinobacteria, (C) Tenericutes, (D) Spirochaetes, (E) Proteobacteria, and (F) Euryarchaeota. Data are presented as means \pm SEM $(\mathrm{n}=6)$. Con. = control, LZM $150=$ control diet + lysozyme $150 \mathrm{mg} / \mathrm{kg}$, LZM $300=$ control diet + lysozyme $300 \mathrm{mg} / \mathrm{kg}$. A, B, significant effect of treatment (values with different uppercase letters are significantly different, $P<0.01$ ), and a, b significant effect of treatment (values with different lowercase letters are significantly different, $P<0.05$ ).

\begin{tabular}{|c|c|c|c|c|c|}
\hline \multirow[b]{2}{*}{ Genera } & \multicolumn{3}{|l|}{ Treatment } & \multirow[b]{2}{*}{$P$-value } & \multirow[b]{2}{*}{ FDR } \\
\hline & Control & LZM 150 & LZM 300 & & \\
\hline \multicolumn{6}{|l|}{ d 1 of lactation } \\
\hline Ruminococcaceae_UCG-005 & $5.15 \pm 0.52^{\mathrm{a}}$ & $2.97 \pm 0.18^{\mathrm{b}}$ & $3.80 \pm 0.36^{\mathrm{b}}$ & 0.008 & 0.015 \\
\hline Ruminococcaceae_UCG-010 & $1.40 \pm 0.09^{\mathrm{a}}$ & $1.26 \pm 0.08^{\mathrm{ab}}$ & $1.06 \pm 0.09^{\mathrm{b}}$ & 0.049 & 0.052 \\
\hline Romboutsia & $0.23 \pm 0.03^{\mathrm{b}}$ & $0.23 \pm 0.03^{\mathrm{b}}$ & $0.34 \pm 0.03^{\mathrm{a}}$ & 0.031 & 0.040 \\
\hline Acinetobacter & $0.57 \pm 0.32^{\mathrm{b}}$ & $0.35 \pm 0.25^{\mathrm{b}}$ & $2.63 \pm 0.53^{\mathrm{a}}$ & 0.005 & 0.019 \\
\hline \multicolumn{6}{|l|}{ d 7 of lactation } \\
\hline Parabacteroides & $0.59 \pm 0.10^{\mathrm{a}}$ & $0.27 \pm 0.05^{\mathrm{b}}$ & $0.32 \pm 0.09^{\mathrm{b}}$ & 0.033 & 0.040 \\
\hline Prevotella_1 & $0.24 \pm 0.04^{\mathrm{a}}$ & $0.12 \pm 0.04^{\mathrm{b}}$ & $0.06 \pm 0.01^{\mathrm{b}}$ & 0.007 & 0.016 \\
\hline Ruminococcaceae_UCG-005 & $4.36 \pm 0.49^{\mathrm{ab}}$ & $5.43 \pm 0.54^{\mathrm{a}}$ & $3.17 \pm 0.24^{\mathrm{b}}$ & 0.008 & 0.015 \\
\hline Ruminococcaceae_UCG-014 & $2.55 \pm 0.61^{\mathrm{a}}$ & $1.34 \pm 0.20^{\mathrm{ab}}$ & $0.86 \pm 0.11^{\mathrm{b}}$ & 0.018 & 0.028 \\
\hline Cellulosilyticum & $0.46 \pm 0.06^{\mathrm{a}}$ & $0.29 \pm 0.04^{\mathrm{b}}$ & $0.24 \pm 0.03^{\mathrm{b}}$ & 0.005 & 0.023 \\
\hline Ruminococcaceae_UCG-009 & $0.27 \pm 0.04^{\mathrm{b}}$ & $0.32 \pm 0.04^{\mathrm{ab}}$ & $0.42 \pm 0.04^{\mathrm{a}}$ & 0.047 & 0.049 \\
\hline Succinivibrio & $0.19 \pm 0.07^{\mathrm{a}}$ & $0.05 \pm 0.01^{\mathrm{b}}$ & $0.04 \pm 0.01^{\mathrm{b}}$ & 0.041 & 0.045 \\
\hline \multicolumn{6}{|l|}{ d 21 of lactation } \\
\hline Methanobrevibacter & $1.42 \pm 0.52^{\mathrm{b}}$ & $4.47 \pm 1.60^{\mathrm{a}}$ & $0.34 \pm 0.18^{\mathrm{b}}$ & 0.025 & 0.036 \\
\hline Lactobacillus & $1.28 \pm 0.45^{\mathrm{b}}$ & $6.05 \pm 1.56^{\mathrm{a}}$ & $3.86 \pm 0.56^{\mathrm{a}}$ & 0.014 & 0.023 \\
\hline Terrisporobacter & $3.71 \pm 0.17^{\mathrm{a}}$ & $3.32 \pm 0.25^{\mathrm{a}}$ & $2.06 \pm 0.50^{\mathrm{b}}$ & 0.008 & 0.016 \\
\hline Enterococcus & $1.95 \pm 0.58^{\mathrm{b}}$ & $1.45 \pm 0.50^{\mathrm{b}}$ & $5.65 \pm 0.41^{\mathrm{a}}$ & $<0.001$ & $<0.01$ \\
\hline Christensenellaceae_R-7_group & $2.27 \pm 0.25^{\mathrm{a}}$ & $2.56 \pm 0.16^{\mathrm{a}}$ & $1.41 \pm 0.12^{\mathrm{b}}$ & 0.006 & 0.016 \\
\hline Staphylococcus & $0.74 \pm 0.34^{\mathrm{b}}$ & $0.91 \pm 0.51^{\mathrm{b}}$ & $2.71 \pm 0.18^{\mathrm{a}}$ & 0.004 & 0.012 \\
\hline Oscillospira & $0.77 \pm 0.09^{\mathrm{a}}$ & $0.73 \pm 0.10^{\mathrm{a}}$ & $0.42 \pm 0.09^{\mathrm{b}}$ & 0.031 & 0.041 \\
\hline Ruminococcaceae_UCG-009 & $0.21 \pm 0.05^{\mathrm{b}}$ & $0.42 \pm 0.05^{\mathrm{a}}$ & $0.31 \pm 0.04^{\mathrm{ab}}$ & 0.014 & 0.024 \\
\hline Ruminococcaceae_UCG-010 & $1.32 \pm 0.10^{\mathrm{a}}$ & $1.03 \pm 0.10^{\mathrm{ab}}$ & $0.95 \pm 0.08^{\mathrm{b}}$ & 0.035 & 0.040 \\
\hline Ruminococcaceae_UCG-013 & $0.20 \pm 0.01^{\mathrm{b}}$ & $0.26 \pm 0.03^{\mathrm{a}}$ & $0.26 \pm 0.03^{\mathrm{a}}$ & $<0.001$ & $<0.01$ \\
\hline Family_XIII_AD3011_group & $0.65 \pm 0.07^{\mathrm{ab}}$ & $0.83 \pm 0.04^{\mathrm{a}}$ & $0.40 \pm 0.04^{\mathrm{b}}$ & $<0.001$ & $<0.01$ \\
\hline Romboutsia & $0.31 \pm 0.02^{\mathrm{a}}$ & $0.22 \pm 0.03^{\mathrm{ab}}$ & $0.13 \pm 0.03^{\mathrm{b}}$ & 0.007 & 0.020 \\
\hline
\end{tabular}

Table 3. The relative abundances at genus level $(\%,>0.1 \%$ in at least one sample) of feeding sows diets supplemented with lysozyme. Data are expressed as mean $\pm \mathrm{SD}$. Sows were regarded as the experimental units, $\mathrm{n}=6$ for each treatment. LZM $150=$ control diet + lysozyme $150 \mathrm{mg} / \mathrm{kg}, \mathrm{LZM} 300=$ control diet + lysozyme $300 \mathrm{mg} / \mathrm{kg}$. ${ }^{\mathrm{a} b \mathrm{~b}}$ Within a row, means with different superscripts are different $(P<0.05)$. 
A

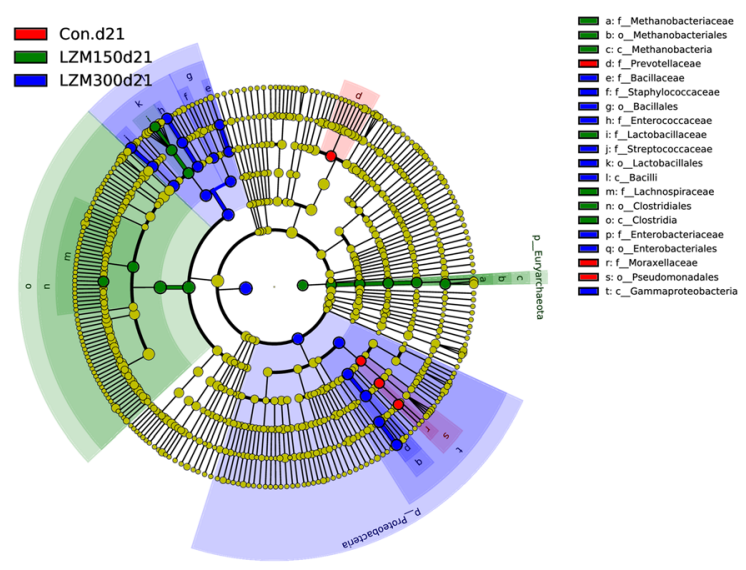

B

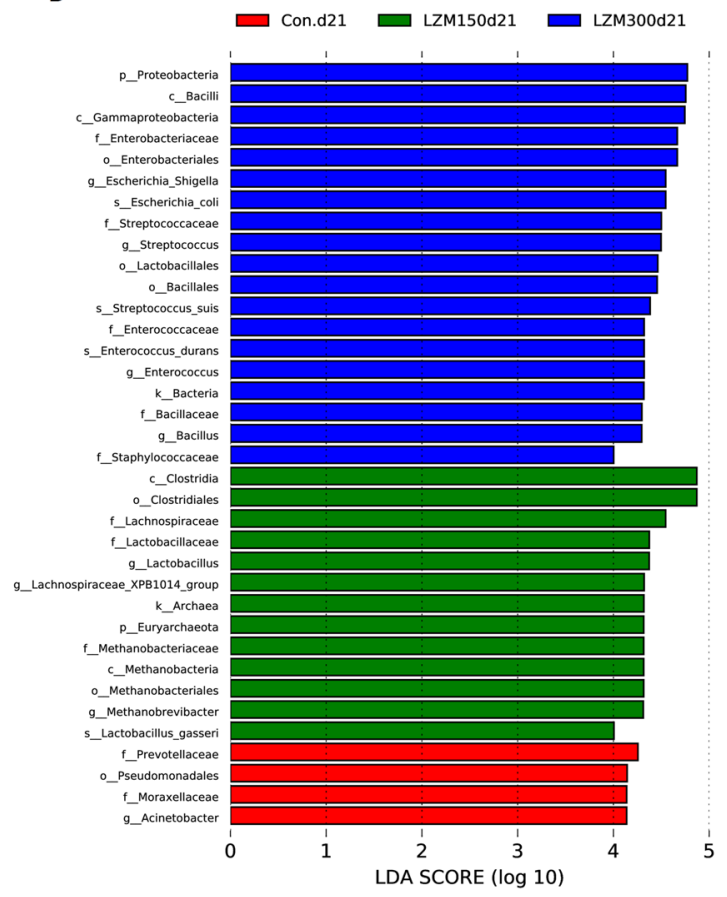

Figure 5. LEfSe analysis of the gut microbiota composition on $\mathrm{d} 21$ of lactation of feeding sows diets supplemented with lysozyme. (A) Cladogram using LEfSe method indicating the phylogenetic distribution of gut microbiota in sows supplemented with lysozyme. Each successive circle represents a phylogenetic level. (B) Histogram of the LDA scores reveals the most differentially abundant taxa among different treatments. Con. = control, LZM $150=$ control diet + lysozyme $150 \mathrm{mg} / \mathrm{kg}$, LZM $300=$ control diet + lysozyme $300 \mathrm{mg} / \mathrm{kg}$.

\section{Discussion}

Lysozyme lyses a specific link in the peptidoglycan layer of bacterial cell walls resulting in cell decomposition ${ }^{23}$. It has been reported that piglets which consume LZM that had been highly expressed in transgenic goats' milk showed decreased Firmicutes and increased Bacteroidetes in their feces ${ }^{25}$. The abundant phyla Firmicutes and Bacteroidetes accounted for $85 \%$ of microbiota in the sow feces in this study, which is in good agreement with previous studies on pigs $^{26,27}$. It was suggested by one study that increased Firmicutes means the body has a higher capacity for energy acquisition from their diets ${ }^{28}$. However, no difference was found between the Firmicutes and the ratio of Firmicutes/Bacteroidetes in feces depending on the lysozyme treatment. The difference between these studies may be due to differing lysozyme types, dosages, and physiological stages of pigs along with differences in the duration of feeding. Zou et al. ${ }^{24}$ found that adding $100 \mathrm{mg} / \mathrm{kg}$ lysozyme (L100) to the diets of growing pig decreased cecal microflora evenness compared with control or L50 groups through 16S rRNA sequencing. In this study, we found microbial diversity decreased with lysozyme supplementation only in the LZM 300 treatment on day 21 of lactation. In addition, no difference was found in the richness of microbiota community between treatments. Interestingly, we also found that the microbiota community richness was increased with the lactation progress. This is in agreement with Cheng et al. ${ }^{8}$, who found the lowest gut microbial richness on day 3 of lactation. This may help explain how the physiological process of farrowing significantly changes the abundance of maternal gut microbiota, as well as how microflora continue to remodel and stabilize over the time.

Fermentation of dietary fiber by microbiota in the hindgut produces many SCFAs, including acetic acid, propionic acid and butyric acid ${ }^{29}$. With intestinal absorption, acetate is most frequently used for lipid biosynthesis ${ }^{30}$. Propionate tends to be a precursor of gluconeogenesis ${ }^{31}$. Butyrate, mainly used as an energy source, is metabolized by colonocytes and regulates the growth and death of both epithelial and immune cells $\mathrm{s}^{32,33}$. In addition, SCFAs are signal molecules for regulating lipid and glucose metabolism by combining with free fatty acid receptors $^{34,35}$. These contribute to the key role of SCFA in the diet-gut microbiome-host metabolism axis. Acetate, a carbohydrate fermentation product, is produced by the majority of enteric bacteria. It was more than $50 \%$ of the total SCFA in feces in this study, which is in agreement with the findings of both Zhou et al. ${ }^{2}$ and Cheng et al. ${ }^{8}$.

Additionally, we found that concentrations of total and individual SCFA increased from day 1 to day 21 of lactation, which aligns with the work of Cheng et al. ${ }^{8}$, who found an increase in butyrate from day 3 to day 14 in lactating sows. Diets supplemented with lysozyme decreased sows' acetate, butyrate and SCFAs on day 7 of lactation, while they decreased the butyrate on day 21 of lactation compared with the control treatment. Based on our previous data, sows on lysozyme diets didn't have any difference in back fat, weight increase during the gestation period or weight loss during the lactation period when compared with sows in the control treatment ${ }^{22}$. It seems that the decrease of the SCFAs didn't affect sows' lipid and glucose metabolism. Higher SCFAs in stool may indicate a greater amount of energy loss in feces, as suggested by Koren et al. ${ }^{36}$. The SCFAs 


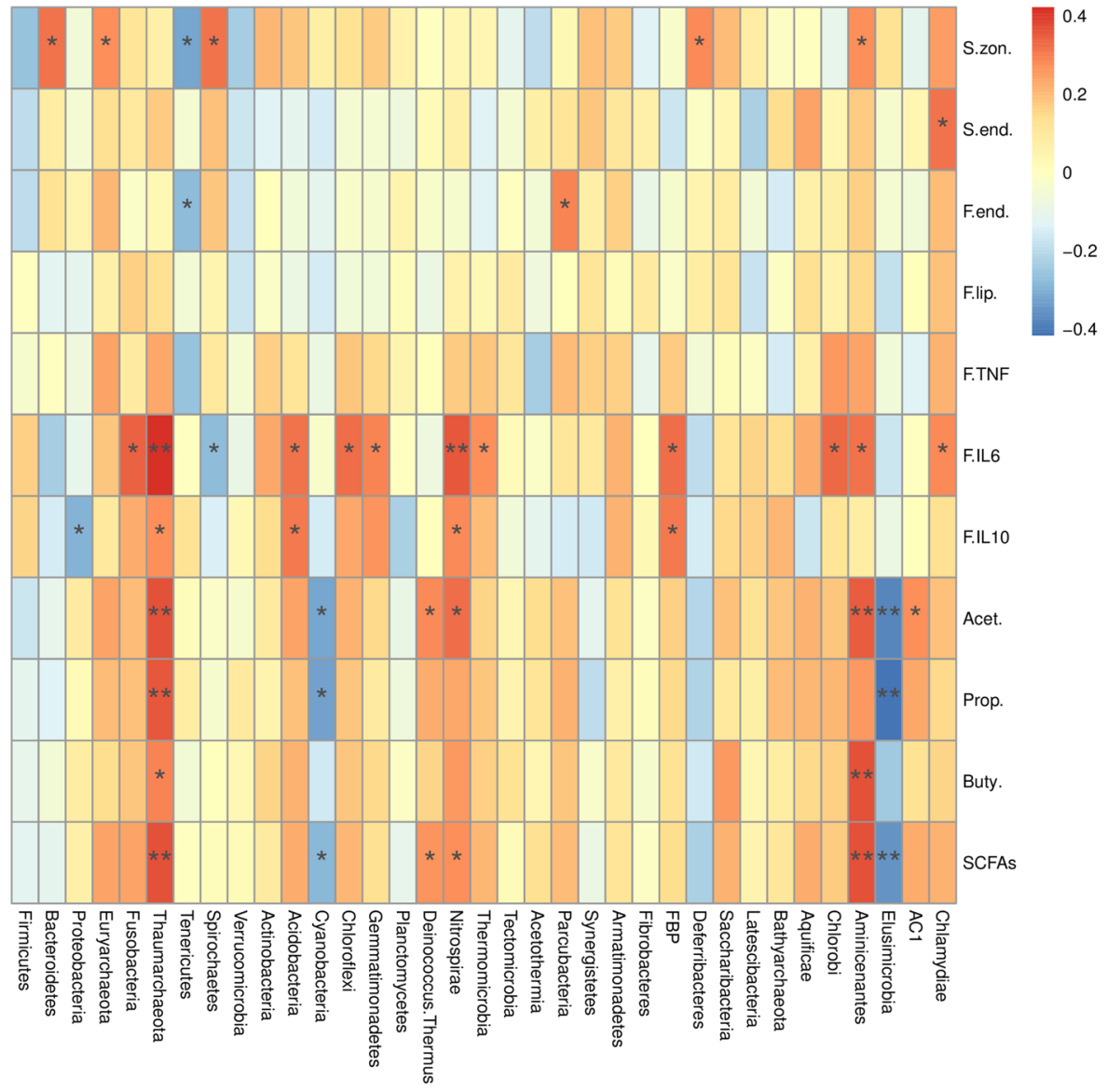

Figure 6. Heatmap of the spearman $r$ correlations between the gut microbiota (phylum level) significantly modified by metabolic parameters of sows. Data are presented as means $\pm \operatorname{SEM}(\mathrm{n}=6) . * P<0.05 ; * * P<0.01$ (following the Spearman correlation analysis). S.zon. = serum_zonulin, S.end. = serum endotoxin, F.end = fecal endotoxin, F.lip. $=$ fecal lipocalin-2, F.TNF $=$ fecal TNF- $\alpha$, F.IL6 $=$ fecal IL-6, F.IL10 = fecal IL-10, Acet. $=$ acetate, Prop. = propionate, Buty. = butyrate, SCFAs is the sum of acetate, propionate, and butyrate.

had higher colonic absorption, with less than $5 \%$ being excreted in feces in humans ${ }^{37}$. When compared to production or absorption of SCFAs per se, fecal SCFA concentrations are more suitable for reflecting the overall net production and absorption of SCFAs in gastrointestinal tracts ${ }^{38,39}$. In order to assess SCFAs potential production, SCFA producing-related phylum and genera were analyzed. At the phylum level, Bacteroides, which mainly produces propionate, was decreased by LZM supplementation at $300 \mathrm{mg} / \mathrm{kg}$ on day $7 \mathrm{of} \mathrm{lactation}$. The concentration of propionate had a tendency $(P=0.06)$ to decrease with LZM supplementation on that day. Despite the butyrate-producing genus Romboutsia being significantly decreased, Lactobacillus was significantly increased by the addition of LZM on day 21 of lactation. This was consistent with previous studies, which found lysozyme is beneficial to enrich Lactobacilli in the intestine of breastfed infants $\mathrm{s}^{10-12}$. This possibly means that LZM supplementation in this study decreased SCFAs-producing genera and decreased the SCFAs. However, we did not find any changes in the related phenotypic indicators. This may be attributed to the reduction of SCFAs, which had not reached the threshold that causes phenotypic changes.

The increase in zonulin concentrations in blood may reflect the impaired intestinal mucosal integrity ${ }^{40}$. It is also the case that low intestinal microorganism richness has been associated with increased intestinal permeability in overweight pregnant women ${ }^{41}$. In this study, we observed that sows which had been fed LZM 150 and 300 diets had both decreased levels of zonulin in their serum on days 7 and 21 of lactation, but although no difference was found on day 1 of lactation. These findings indicate that the gut permeability of sows decreased with the addition of lysozyme suggesting lysozyme may modulate the intestinal barrier integrity in sows. While microbiota community richness was increased by the lactation progress, we didn't observe a decrease in gut permeability in late lactation. This may due to the differences between human and sows, or could be because the microbiota richness variety hasn't reached a threshold which induces changes in gut permeability. Correlation analysis at the phylum level found that Bacteroidetes and Spirochaetes were positively correlated with serum zonulin. The $16 \mathrm{~s}$ rRNA results found that LZM reduced the abundance of Bacteroidetes and Spirochaetes, meaning that serum zonulin would decrease. Although Tenericutes was negatively correlated with serum zonulin, and the results of $16 \mathrm{~s}$ rRNA found that LZM reduced the abundance of Tenericutes, meaning that the concentration of serum zonulin may increase, but this was not consistent with the detected results. The above results indicate that there are many influencing factors to affect serum zonulin, and the change in its concentration was a result of multiple factors. 
At the genus level, consistent with a zonulin concentration decrease in serum, sows which had been fed LZM 150 and LZM 300 diets had decreased Cellulosilyticum on day 7 of lactation, while sows which had been fed LZM 300 diets had increased Staphylococcus on day 21 of lactation. Spearman's correlation found that Cellulosilyticum was positively correlated with serum zonulin, while Staphylococcus was negatively correlated with the serum zonulin (Fig. S5B). This possibly meant that sows which had been fed LZM diets had decreased phylum Bacteroidetes and Spirochaetes, genus Cellulosilyticum and increased Staphylococcus, resulting in a decrease in zonulin concentration, which in turn reflects a decline in gut permeability. Additional studies should be performed in order to demonstrate this hypothesis.

It also is reported that butyrate maintains an intestinal barrier integrity via a modulation of expression of tight junction proteins ${ }^{42,43}$. However, we found that sows which had been fed LZM 150 and LZM 300 diets both had a decreased concentration of butyrate and decreased endotoxin concentration in serum and feces. Endotoxin generally refers to lipopolysaccharide (lipid A) and it is a pyrogen, which is mainly present in the bacterial cell wall of gram-negative bacteria ${ }^{44}$. At the phylum level, we found that LZM reduced the gram-negative bacteria Bacteroidetes, but increased the gram-negative bacteria Proteobacteria. However, in terms of the proportion of microorganisms, Bacteroidetes was $11.95 \%$, while Proteobacteria was $6.54 \%$. This may be one important reason why LZM can reduce endotoxin levels in the serum and fecal material. In addition, phylum Spirochaetes decreased with the addition of LZM. One study demonstrated that many of the organisms within the Spirochaetes phylum cause prevalent diseases. Pathogenic members of this phylum include Brachyspira pilosicoli and Brachyspira aalborgi, which both cause intestinal spirochaetosis ${ }^{45}$. Based on these observations, we suggest that while the addition of LZM does not increase the content of butyrate, it reduces intestinal permeability and endotoxin content as well as reducing level of Spirochaetes, which may be beneficial to intestinal health.

At the phylum level, we observed that sows which had been fed LZM 150 and LZM 300 diets decreased Actinobacteria and Tenericutes on days 1 and 21 of lactation. The two kinds of phylum were thought to be associated with inflammation responses. Patients with inflammatory bowel disease ${ }^{46}$ as well as obese Ossabaw minipigs ${ }^{47}$ have high levels of Actinobacteria at the colon. In general, chronic or low-grade inflammation is a hallmark of obesity and metabolic disorders ${ }^{48,49}$. It has also been found that, along with gestational weight gain induced by the addition of fat, the phylum Actinobacteria increased. From the inflammatory indicators they found that the sows were in high-grade inflammation ${ }^{2}$. Similarly, high levels of phylum Tenericutes have been found in diet-induced obese mice ${ }^{50}$ and obese Göttingen pigs $^{47}$. We found that Tenericutes decreased with the addition of LZM. This effect of LZM was similar with the report of Everard et al..$^{51}$ who found that the addition of probiotic yeast decreased the abundance of Tenericutes. Given these observations, we hypothesized that sows fed LZM diets present with low-grade inflammation compared with control. Consistent with this hypothesis, lipocalin-2 and pro-inflammatory cytokines TNF- $\alpha$ in feces were decreased in the LZM 150 and 300 groups, while the IL-10 (anti-inflammatory cytokines) increased in the LZM 150 group on days 7 and 21 of lactation. Consistent with these findings, our previous study found that LZM 150 and LZM 300 increased IL-10 in serum on day 7 of sow lactation, and had a tendency to enhance IL-10 in serum on day 1 of lactation ${ }^{22}$, and previous studies also found that lysozyme are involved in modulating the inflammatory response ${ }^{14,15}$. Cytokines regulate both immune responses $^{52}$ and nutrient distribution which, characterized by proinflammatory cytokines, induce more nutrients to enter the immune response rather than being used for growth ${ }^{53,54}$. Lower TNF- $\alpha$ was found in pigs which had been fed lysozyme diets than in control groups ${ }^{20}$. Phylum Proteobacteria contains multiple pathogens and also has proinflammatory properties ${ }^{55}$. High levels of Proteobacteria are a potential diagnostic feature of epithelial dysfunction and dysbiosis in gut microbiota ${ }^{56,57}$. We found that LZM supplementation at $300 \mathrm{mg} / \mathrm{kg}$ enriched the Proteobacteria on day 1 of lactation. However, we didn't find any cytokine differences between treatments. Based on these findings, we propose that pro-inflammatory or anti-inflammatory factors are affected by changes in microbial flora; not only by a single microbe, but by the results of multiple microbial interactions. However, the possible mechanisms for how gut microbiota interact to cause intestinal inflammation in perinatal period maternal body require further study.

In conclusion, this study suggested that gut microbiota and their metabolites were altered in sows following the addition of LZM to their diets during the period of late gestation to lactation. Typical changes involve the expansion of diversity among treatments, an increase in Lactobacillus as well as a decrease in Romboutsia, Spirochaetes, Actinobacteria and Tenericutes in LZM 300 treatment. Microbial changes triggered by the additional of LZM during late gestation to lactation could potentially be the mechanism for the positive effects seen of LZM on gut permeability and gut health regulation, as well as maternal anti-inflammation. Until now, there has been little knowledge about the maternal microbial alterations induced by LZM supplementation, meaning that special attention should be given to this area in future research. Using lysozyme in maternal diets from late gestation to lactation may be a beneficial way of preventing gut permeability, related inflammation status and metabolites disturbances with a remodeled microbial ecosystem.

\section{Materials and Methods}

All animal procedures in this study were approved by the Animal Care and Use committee of Sichuan Agricultural University, under ethical approval number DKY-S20156137. All the experiments were performed in accordance with the guidelines and regulations of the Animal Care and Ethical Committee of the Sichuan Agricultural University.

Animals and experimental design. A total of 60 Yorkshire $\times$ Landrace sows (3-6 parity, $14.07 \pm 2.58 \mathrm{~mm}$ backfat thickness) were used in this study, which was designed as a single factorial arrangement of treatments including the control group (basal diet, $n=20$ ), LZM 150 diet group (basal diet $+150 \mathrm{mg} / \mathrm{kg}$ lysozyme, concentration in accordance with the results of antibacterial tests, data not shown, $n=20$ ), and the LZM 300 diet group (basal diet $+300 \mathrm{mg} / \mathrm{kg}$ lysozyme, $n=20$ ). The sows received treatment diets from day 85 of gestation 
(usually farrowing at day 114 of gestation) to the end of weaning (day 21 of lactation). Based on a corn-soybean meal, the diet was formulated to meet nutritional requirements recommended by the National Research Council 2012 (NRC 2012). The gestation basal diet included $3.04 \mathrm{Mcal}$ digestible energy (DE)/kg, 14.65\% of crude protein, $0.69 \%$ Lys, $0.85 \%$ calcium, and $0.67 \%$ phosphorus, while the lactation basal diet included $3.29 \mathrm{Mcal} \mathrm{DE} / \mathrm{kg}$, $17.54 \%$ of crude protein, $0.99 \%$ Lys, $0.99 \%$ calcium and $0.68 \%$ phosphorus. None of the diets included antibiotics, probiotics or other medicines. The LZM used in the study was given by Shanghai Longyou Biotechnology Co., Ltd. Enzyme activity is $5000 \mathrm{U} / \mathrm{mg}$.

During gestation (day 85 to 106), all sows were housed in individual gestation stalls. On day 107 of pregnancy, sows were transferred to individual farrowing crates. Before farrowing, sows were fed an average of $3.5 \mathrm{~kg} / \mathrm{d}$ diet. On farrowing days, sows were fed $0.5 \mathrm{~kg}$ of feed, which was gradually increased by $1.0 \mathrm{~kg} / \mathrm{d}$ until the maximum feeding amount was reached as described as before ${ }^{22}$. Sows were then allowed to feed ad libitum throughout the rest of their lactation days. Sows had free access to water in this study.

Sample collection. On day 1 of lactation, 10 sows were randomly selected from each treatment. Used the same sows to collect samples at days 7 and 21 of lactation. Fresh feces were collected by massaging the rectum of each sow ${ }^{8}$. Sows had no diarrhea or other disease before sampling. On day 1, samples were collected within two hours after farrowing. Fresh feces were stored in three sterile tubes and kept in liquid nitrogen before being transferred to $-80^{\circ} \mathrm{C}$. The $5 \mathrm{~mL}$ blood sample was obtained from the same 10 sows' ear veins for each treatment group before morning meal on days 1,7 and 21 of lactation. Serum samples were obtained by centrifuging blood for $10 \mathrm{~min}$ at $3,000 \mathrm{~g}$ at $4{ }^{\circ} \mathrm{C}$, and were then stored at $-20^{\circ} \mathrm{C}$ for further analysis ${ }^{22}$.

Analysis of fecal pH value and SCFAs. Fecal $\mathrm{pH}$ values $(\mathrm{n}=10)$ were detected following the method of Topping et al. ${ }^{58}$. Briefly, distilled water was used to dilute $0.5 \mathrm{~g}$ of feces at a ratio of 1:2 (weight/volume) by a blender. Fecal homogenate was then centrifuged $\left(20^{\circ} \mathrm{C}\right)$ for $15 \mathrm{~min}$ at $3,000 \mathrm{~g}$, after which the supernatant was measured by $\mathrm{pH}$ meter ${ }^{58}$ (PHS-3C $\mathrm{pH}$, Shanghai, China).

Concentrations of the fecal SCFAs (acetate, propionate and butyrate, $\mathrm{n}=10$ ) were analyzed as described by Chen et al. ${ }^{59}$. A $2 \mathrm{~g}$ feces sample was suspended in distilled water $(5 \mathrm{~mL})$ and allowed to stand for $30 \mathrm{~min}$, then it was centrifuged for $10 \mathrm{~min}$ at $12,000 \mathrm{~g}$ at $4^{\circ} \mathrm{C}$. The $2 \mathrm{~mL}$ of sample supernatant was then transferred and mixed with $0.4 \mathrm{~mL}$ of metaphosphoric acid. The sample placed at $4{ }^{\circ} \mathrm{C}$ for $30 \mathrm{~min}$, and centrifuged again for $10 \mathrm{~min}$ at $12,000 \mathrm{~g}$ at $4^{\circ} \mathrm{C}$ before being transferred to $1.2 \mathrm{~mL}$ of supernatant and mixed with $15.2 \mu \mathrm{L}$ of crotonic acid $\left(210 \mathrm{mmol} / \mathrm{L}\right.$, internal standard), after which $0.3 \mathrm{~mL}$ liquid was mixed with $0.3 \mathrm{~mL}$ methanol ${ }^{59}$. Finally, $1 \mu \mathrm{L}$ of supernatant was used to analyze of SCFAs by a gas chromatograph ${ }^{59}$ (GC, Varian CP-3800 GC, USA; capillary column $30 \mathrm{~m} \times 0.32 \mathrm{~mm} \times 0.25 \mu \mathrm{m}$ film thickness). The minimal detection limit for each SCFA was $0.1 \mathrm{mmol} / \mathrm{L}$.

Analysis of metabolic biomarker. The gut health related metabolic biomarkers lipocalin-2, endotoxin, interleukin-6 (IL-6), tumor necrosis factor- $\alpha$ (TNF- $\alpha$ ), interleukin-10 (IL-10) of fecal as well as zonulin and endotoxin of serum $(n=8,8$ samples were randomly selected from the 10 sows of which were randomly selected for the sample collection of each treatment at day 1 of lactation. Samples came from the same 8 sows of days 7 and 21 of lactation of each treatment.) were measured using porcine enzyme-linked immunosorbent assay (ELISA) Kits (R\&D Systems Inc., Minneapolis, MN, USA). The biomarker analysis was performed according to the manufacturer's instructions.

Analysis of bacterial community. The Mo Bio PowerFecal ${ }^{\mathrm{TM}}$ DNA Isolation Kit (MO BIO Laboratories, Carlsbad, CA, USA) was used to extract the microbial DNA of thawed fecal sample $(0.5 \mathrm{~g}, \mathrm{n}=6,6$ samples were randomly selected from the 8 sows of which were randomly selected for the metabolic biomarker analysis of each treatment at day 1 of lactation. Samples came from the same 6 sows of days 7 and 21 of lactation of each treatment.). The nucleic acid/protein analyzer (Beckman DU-800, Beckman Coulter, Inc., CA, USA) was utilized to examine the concentration and purity of DNA. Then the DNA samples were sent to perform amplicon pyrosequencing on Illumina HiSeq PE250 platforms and bioinformatics analyses at Novogene Bioinformatics Technology in Beijing, China. The V4 hypervariable region of the $16 \mathrm{~S}$ rRNA gene was amplified using a forward primer $515 \mathrm{f}$ (5'-GTGCCAGCMGCCGCGGTAA-3') and a reverse primer 806r (5'-GGACTACHVGGGTWTCTAAT- $\left.{ }^{\prime}\right)$ as described as before ${ }^{2}$.

The linker sequence, barcode sequence in the reads, homopolymer runs exceeding $6 \mathrm{bp}$, primer mismatches, and sequence lengths shorter than $100 \mathrm{bp}$ were removed. Paired-end reads from the original DNA fragments were merged using FLASH version 1.2.760. Then Quantitative Insights Into Microbial Ecology ${ }^{61}$ (QIIME) version 1.7.0. was applied to filter out sequences containing ambiguous bases $(\mathrm{N})$ or low-quality bases of the merging data. Following removed the chimeric sequences in the splicing sequence. High-quality tags were clustered into OTUs utilizing Uparse v7.0.1001 (http://drive5.com/uparse/) at 97\% sequence similarity. The Ribosomal Database Project (RDP) classifier Version 2.2 (http://github.com/rdpstaff/) was applied to assign taxonomy for 16S rRNA gene sequences. The relative abundance of each OTU was examined at different taxonomic levels. A Venn diagram was generated for comparison among the OTUs of the three treatments. Alpha diversity values for each sample were assessed by Qiime 1.7.0. To identify bacterial taxa differentially represented among different treatments at genus or higher taxonomy levels, the linear discriminant analysis coupled with effect size (LEfSe) was applied ${ }^{62}$.

Statistical analysis. The original data were checked using Grubbs' test method. If $\left|X p-{ }^{-} X\right| a ̊ n(\alpha, n) S$, the $\mathrm{Xp}$ was considered as the outlier. Before using parametric analyses, descriptive statistics were performed to check the normality and homogeneity of variances. Data of relative abundance at the phylum and genus level in fecal matter were log-transformed before statistical analysis. The data were analyzed using the General Linear Model (GLM) procedures of SAS (V9.3, SAS Institute Inc., Cary, NC, USA) followed by a DUNCAN analysis for 
multiple comparison when the $\mathrm{F}$ test in the analysis of variance table was significant. The statistical model was: $Y_{i j}=\mu+t_{i}+e_{i j}$ where $Y i j$ is the analyzed variable, $\mu$ is the overall mean, $t$ is the effect of treatment $\left({ }_{i}=1,2,3\right)$, and $e$ is the residual error $\left({ }_{i}=1,2,3,{ }_{j}=1 \ldots 10\right.$ or 6$)$. Normality of residual check was performed using PROC UNIVARIATE with Normal and Plot options in SAS. Data for the figures (Figs. 1,2,4, S1) were analyzed using a one-way ANOVA followed by Tukey's test for multiple comparisons using GraphPad Prism analysis software. All data are shown as mean \pm SEM. Differences of $p<0.05$ were considered significant when, whereas $p<0.10$ was considered a trend.

To analyze correlations between microbiota and metabolic parameters, Spearman's correlation in R 3.0.2 with the Rstudio 0.97.310 package and gplots package for the heat map were used. Differences of $p<0.05$ were considered significant. Data were corrected by false discovery rate analysis according to the Benjamini-Hochberg method with an $\alpha$ of $<0.05^{63}$.

Received: 24 July 2019; Accepted: 4 February 2020;

Published online: 21 February 2020

\section{References}

1. Newbern, D. \& Freemark, M. Placental hormones and the control of maternal metabolism and fetal growth. Current Opinion in Endocrinology, Diabetes and Obesity 18, 409-416 (2011).

2. Zhou, P. et al. Microbial mechanistic insight into the role of inulin in improving maternal health in a pregnant sow model. Frontiers in Microbiology 8, 2242 (2017).

3. Fung, T. C., Olson, C. A. \& Hsiao, E. Y. Interactions between the microbiota, immune and nervous systems in health and disease. Nature Neuroscience 20, 145-155 (2017).

4. Belkaid, Y. \& Harrison, O. J. Homeostatic immunity and the microbiota. Immunity. 46, 562-576 (2017).

5. Ervin, S. M. et al. Gut microbiome-derived $\beta$-glucuronidases are components of the estrobolome that reactivate estrogens. Journal of Biological Chemistry 294, 18586-18599 (2019).

6. Kaiser, M. et al. Inflammatory markers before and after farrowing in healthy sows and in sows affected with postpartum dysgalactia syndrome. BMC Veterinary Research 14, 83 (2018).

7. Pomorska-Mól, M., Wierzchosławski, K., Włodarek, J., Gogulski, M. \& Pejsak, Z. Dynamics of pro- and anti-inflammatory cytokine changes in serum and assessment of their diagnostic utility during lactation impairment in pigs. BMC Veterinary Science 128, 9-15 (2019).

8. Cheng, C. et al. Metabolic syndrome during perinatal period in sows and the link with gut microbiota and metabolites. Frontiers in Microbiology 9, 1989 (2018).

9. Chandan, R. C., Parry, R. M. \& Shahani, K. M. Lysozyme, lipase, and ribonuclease in milk of various species. Journal of Dairy Science 51, 606-607 (1968)

10. Coppa, G. V., Zampini, L., Galeazzi, T. \& Gabrielli, O. Prebiotics in human milk: a review. Digestive and Liver Disease 38((Suppl 2)), S291-S294 (2006).

11. Newburg, D. S. \& Walker, W. A. Protection of the neonate by the innate immune system of developing gut and of human milk. Pediatric Research 61, 2-8 (2007).

12. Solis, B. et al. Probiotics as a help in children suffering from malnutrition and diarrhoea. European Journal of Clinical Nutrition 56((Suppl 3)), S57-S59 (2002).

13. Schiffrin, E. J. \& Blum, S. Interactions between the microbiota and the intestinal mucosa. European Journal of Clinical Nutrition 56((Suppl 3)), S60-S64 (2002).

14. Ginsburg, I. Role of lipoteichoic acid in infection and inflammation. The Lancet Infectious Diseases 2, 171-179 (2002).

15. Muller, C. A., Autenrieth, I. B. \& Peschel, A. Innate defenses of the intestinal epithelial barrier. Cellular and Molecular Life Sciences 62, 1297-1307 (2005).

16. May, K. D., Wells, J. E., Maxwell, C. V. \& Oliver, W. T. Granulated lysozyme as an alternative to antibiotics improves growth performance and small intestinal morphology of 10-day-old pigs. Journal of Animal Science 90, 1118-1125 (2012).

17. Oliver, W. T. \& Wells, J. E. Lysozyme as an alternative to antibiotics improves growth performance and small intestinal morphology in nursery pigs. Journal of Animal Science 91, 3129-3136 (2013).

18. Nyachoti, C. M., Kiarie, E., Bhandari, S. K., Zhang, G. \& Krause, D. O. Weaned pig responses to Escherichia coli K88 oral challenge when receiving a lysozyme supplement. Journal of Animal Science 90, 252-260 (2012).

19. Cooper, C. A., Garas Klobas, L. C., Maga, E. A. \& Murray, J. D. Consuming transgenic goats' milk containing the antimicrobial protein lysozyme helps resolve diarrhea in young pigs. PLoS one 8, e58409 (2013).

20. Oliver, W. T., Wells, J. E. \& Maxwell, C. V. Lysozyme as an alternative to antibiotics improves performance in nursery pigs during an indirect immune challenge. Journal of Animal Science 92, 4927-4934 (2014).

21. Long, Y. et al. Effects of dietary lysozyme levels on growth performance, intestinal morphology, non-specific immunity and mRNA expression in weanling piglets. Animal Science Journal 87, 411-418 (2016).

22. Xu, S. et al. Effects of dietary supplementation with lysozyme during late gestation and lactation stage on the performance of sows and their offspring. Journal of Animal Science 96, 4768-4779 (2018).

23. Callewaert, L. \& Michiels, C. W. Lysozymes in the animal kingdom. Journal of Biosciences 35, 127-160 (2010).

24. Zou, L. et al. Effects of dietary lysozyme levels on growth performance, intestinal morphology, immunity response and microbiota community of growing pigs. Journal of the Science of Food and Agriculture 99, 1643-1650 (2019).

25. Maga, E. A. et al. Consumption of lysozyme-rich milk can alter microbial fecal populations. Applied and Environmental Microbiology 78, 6153-6160 (2012).

26. Kong, X. F. et al. Colonic luminal microbiota and bacterial metabolite composition in pregnant Huanjiang mini-pigs: Effects of food composition at different times of pregnancy. Scientific Reports 6, 37224 (2016).

27. Yan, H. et al. Gut microbiota can transfer fiber characteristics and lipid metabolic profiles of skeletal muscle from pigs to germ-free mice. Scientific Reports 6, 31786 (2016).

28. Turnbaugh, P. J. et al. An obesity-associated gut microbiome with increased capacity for energy harvest. Nature 444, 1027-1031 (2006).

29. Rios-Covian, D. et al. Intestinal short chain fatty acids and their link with diet and human health. Frontiers in Microbiology 7,185 (2016).

30. Bloemen, J. G. et al. Short chain fatty acids exchange across the gut and liver in humans measured at surgery. Clinical Nutrition $\mathbf{2 8}$, 657-661 (2009).

31. Roy, C. C., Kien, C. L., Bouthillier, L. \& Levy, E. Short-chain fatty acids: Ready for prime time? Nutrition in Clinical Practice 21, 351-366 (2006).

32. De Vadder, F. et al. Microbiota-generated metabolites promote metabolic benefits via gut-brain neural circuits. Cell 156, $84-96$ (2014). 
33. Pryde, S. E., Duncan, S. H., Hold, G. L., Stewart, C. S. \& Flint, H. J. The microbiology of butyrate formation in the human colon. FEMS Microbiology Letters 217, 133-139 (2002).

34. Brown, A. J. et al. The Orphan G protein-coupled receptors GPR41 and GPR43 are activated by propionate and other short chain carboxylic acids. Journal of Biological Chemistry 278, 11312-11319 (2003).

35. den Besten, G. et al. The role of short-chain fatty acids in the interplay between diet, gut microbiota, and host energy metabolism. The Journal of Lipid Research 54, 2325-2340 (2013).

36. Koren, O. et al. Host remodeling of the gut microbiome and metabolic changes during pregnancy. Cell 150, 470-480 (2012).

37. Raninen, K., Lappi, J., Mykkänen, H. \& Poutanen, K. Dietary fiber type reflects physiological functionality: Comparison of grain fiber, inulin, and polydextrose. Nutrition Reviews 69, 9-21 (2011).

38. Vogt, J. A. \& Wolever, T. M. Fecal acetate is inversely related to acetate absorption from the human rectum and distal colon. The Journal of Nutrition 133, 3145-3148 (2003).

39. Montoya, C. A., Rutherfurd, S. M. \& Moughan, P. J. Kiwifruit fibre level influences the predicted production and absorption of SCFA in the hindgut of growing pigs using a combined in vivo-in vitro digestion methodology. British Journal of Nutrition 115, 1317-1324 (2016).

40. Fasano, A. Zonulin and its regulation of intestinal barrier function: the biological door to inflammation, autoimmunity, and cancer. Physiological Reviews 91, 151-175 (2011).

41. Mokkala, K. et al. Gut microbiota richness and composition and dietary intake of overweight pregnant women are related to serum zonulin concentration, a marker for intestinal permeability. The Journal of Nutrition 146, 1694-1700 (2016).

42. Huang, C. et al. Dietary sodium butyrate decreases postweaning diarrhea by modulating intestinal permeability and changing the bacterial communities in weaned piglets. The Journal of Nutrition 145, 2774-2780 (2015).

43. Kelly, C. J. et al. Crosstalk between microbiota-derived short-chain fatty acids and intestinal epithelial HIF augments tissue barrier function. Cell Host \& Microbe 17, 662-671 (2015).

44. Salguero, M. V. et al. Dysbiosis of Gram-negative gut microbiota and the associated serum lipopolysaccharide exacerbates inflammation in type 2 diabetic patients with chronic kidney disease. Experimental and Therapeutic Medicine 18, 3461-3469 (2019).

45. Amat Villegas, I. et al. Colonic spirochetes: an infrequent cause of adult diarrhea. Gastroenterologia Hepatologia (in Spanish). 27, $21-23(2004)$

46. Frank, D. N. et al. Molecular-phylogenetic characterization of microbial community imbalances in human inflammatory bowel diseases. Proceedings of the National Academy of Sciences of the United States of America 104, 13780-13785 (2007).

47. Pedersen, R. et al. Characterisation of gut microbiota in Ossabaw and Göttingen minipigs as models of obesity and metabolic syndrome. PloS one 8, e56612 (2013).

48. Hotamisligil, G. S. Inflammation and metabolic disorders. Nature 444, 860-867 (2006).

49. Saltiel, A. R. \& Olefsky, J. M. Inflammatory mechanisms linking obesity and metabolic disease. Journal of Clinical Investigation 127, $1-4$ (2017).

50. Turnbaugh, P. J., Bäckhed, F., Fulton, L. \& Gordon, J. I. Diet-induced obesity is linked to marked but reversible alterations in the mouse distal gut microbiome. Cell Host \& Microbe 3, 213-223 (2008).

51. Everard, A., Matamoros, S. E. B., Geurts, L., Delzenne, N. M. \& Cani, P. D. Saccharomyces boulardii administration changes gut microbiota and reduces hepatic steatosis, low-grade inflammation, and fat mass in obese and type 2 diabetic $\mathrm{db} / \mathrm{db}$ mice. MBio 5 , e1011-e1014 (2014).

52. Praveena, P. E., Periasamy, S., Kumar, A. A. \& Singh, N. Cytokine profiles, apoptosis and pathology of experimental Pasteurella multocida serotype A1 infection in mice. Research in Veterinary Science 89, 332-339 (2010).

53. Johnson, R. W. Inhibition of growth by pro-inflammatory cytokines: an integrated view. Journal of Animal Science 75, 1244-1255 (1997).

54. Spurlock, M. E. Regulation of metabolism and growth during immune challenge: an overview of cytokine function. Journal of Animal Science 75, 1773-1783 (1997).

55. Mukhopadhya, I., Hansen, R., El-Omar, E. M. \& Hold, G. L. IBD—what role do Proteobacteria play? Nature Reviews Gastroenterology ¿ Hepatology 9, 219-230 (2012).

56. Shin, N. R., Whon, T. W. \& Bae, J. W. Proteobacteria: microbial signature of dysbiosis in gut microbiota. Trends in Biotechnology 33, 496-503 (2015).

57. Litvak, Y., Byndloss, M. X., Tsolis, R. M. \& Bäumler, A. J. Dysbiotic Proteobacteria expansion: a microbial signature of epithelial dysfunction. Current Opinion in Microbiology 39, 1-6 (2017).

58. Topping, D. L. et al. Dietary fat and fiber alter large bowel and portal venous volatile fatty acids and plasma cholesterol but not biliary steroids in pigs. The Journal of Nutrition 123, 133-143 (1993).

59. Chen, H. et al. Dietary fibre affects intestinal mucosal barrier function and regulates intestinal bacteria in weaning piglets. British Journal of Nutrition 110, 1837-1848 (2013).

60. Magoc, T. \& Salzberg, S. L. FLASH: fast length adjustment of short reads to improve genome assemblies. Bioinformatics 27, 2957-2963 (2011).

61. Caporaso, J. G. et al. QIIME allows analysis of high-throughput community sequencing data. Nature Methods 5, 335-336 (2010).

62. Segata, N. et al. Metagenomic biomarker discovery and explanation. Genome Biology 12, 60 (2011).

63. Benjamini, Y. \& Hochberg, Y. On the adaptive control of the false discovery rate in multiple testing with independent statistics. Journal of Educational and Behavioral Statistics 25, 60-83 (2000).

\section{Acknowledgements}

This study was supported by the Application and Demonstration of High-efficiency and Safe Breeding Technology for High-yield Breeding Pigs Project (2018YFD0501005), Sichuan Province “135" Breeding Tackle Project (Project No. 2016NYZ0052), Shanghai Longyou Biotechnology CO. LTD, Sichuan Agricultural University Double Support Project and the 111 Project (D17015). The authors would like to thank Dr. Paul Dyce for his careful editing of this manuscript.

\section{Author contributions}

S.X. contributed to the experimental design. J.S. performed the animal experiments. J.S., Y.D., Z.L. and X.W. executed the lab analysis. J.S., Y.D. and S.X. performed the statistical analysis and wrote the manuscript. Y.L., L.C., J.L., B.F., Z.F., Z.Y., J.W., D.W., Z.R. and Y.S. revised the manuscript. All authors read and approved the final manuscript.

\section{Competing interests}

The authors declare no competing interests. 


\section{Additional information}

Supplementary information is available for this paper at https://doi.org/10.1038/s41598-020-60131-1.

Correspondence and requests for materials should be addressed to S.X.

Reprints and permissions information is available at www.nature.com/reprints.

Publisher's note Springer Nature remains neutral with regard to jurisdictional claims in published maps and institutional affiliations.

(c) (i) Open Access This article is licensed under a Creative Commons Attribution 4.0 International License, which permits use, sharing, adaptation, distribution and reproduction in any medium or format, as long as you give appropriate credit to the original author(s) and the source, provide a link to the Creative Commons license, and indicate if changes were made. The images or other third party material in this article are included in the article's Creative Commons license, unless indicated otherwise in a credit line to the material. If material is not included in the article's Creative Commons license and your intended use is not permitted by statutory regulation or exceeds the permitted use, you will need to obtain permission directly from the copyright holder. To view a copy of this license, visit http://creativecommons.org/licenses/by/4.0/.

(c) The Author(s) 2020 